\title{
Emodialisi quotidiana: strategie logistiche per l'abbattimento dei costi
}

\author{
Jacopo Bartoccini, Valentina Cannizzo \\ Infermiere presso la Struttura Complessa di Nefrologia e Dialisi dell'Ospedale Santa Maria della Misericordia di Perugia, Perugia
}

\begin{abstract}
Daily hemodialysis logistics strategies for cost reduction
Patients treated for end stage renal disease (ESRD) have a shorter life expectancy and a poorer quality of life than the general population. In an attempt to improve outcomes for this population of patients, a few new therapeutic approaches have been undertaken. With hemodialysis, an increase in dialysis frequency and/ or time has been associated to improvements in anemia, left ventricular hypertrophy, hypertension, hyperphosphatemia, nutrition and quality of life. Yet, the access to these promising hemodialysis modalities has remained limited, due to the high cost of the treatments and to the cost to transport patients. This project is going to discuss how to reorganize transport logistics, and shifts of dialysis can give an improvement in quality of life by reducing the comorbidities related to renal failure and dialysis treatment and increasing life expectancy with the reduction of costs.
\end{abstract}

Keywords: Daily hemodialysis, Transport logistics, Quality of life, Nursing care

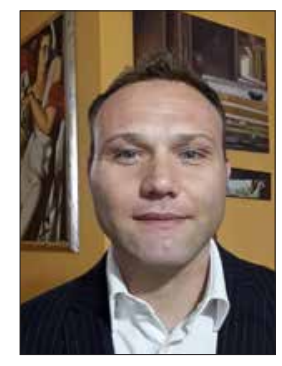

Jacopo Bartoccini

\section{Premessa}

L'emodialisi è un trattamento sostitutivo della funzione renale indispensabile per la sopravvivenza dei pazienti con insufficienza renale cronica; questa viene eseguita, di norma, per 3 volte a settimana, per 4 ore a trattamento, ma vi sono anche altre opzioni che vengono praticate pervia dei benefici che apportano (1):

- $\quad$ emodialisi notturna per 8-10 ore, 6-7 giorni a settimana;

- emodialisi diurna breve, daily hemodialysis (in genere per 2 ore), 6 giorni a settimana;

- emodialisi lunga notturna per 7-8 ore, 3 giorni a settimana (Schema Tassin).

\section{Accepted: July 5, 2015}

Published online: July 23, 2015

\section{Indirizzo per la corrispondenza:}

Jacopo Bartoccini

Infermiere presso la Struttura Complessa di Nefrologia e Dialisi

Ospedale Santa Maria della Misericordia di Perugia

06156 Perugia

jacopobartoccini@libero.it
Un'indagine eseguita nel 2004 ha evidenziato che, globalmente, 1.783 .000 pazienti sono sottoposti a trattamento per la malattia renale all'ultimo stadio (2). L'insufficienza renale cronica costituisce oggi un problema di salute pubblica di primaria importanza per la morbilità e la mortalità significative e per l'elevato impatto socio-economico (3), dovuto al costo dei trattamenti e dei trasporti, ed è per questo che, nella nostra realtà, viene utilizzato lo schema trisettimanale.

Globalmente, la maggior parte dei pazienti con malattia renale cronica che ricevono il trattamento dialitico 3 volte a settimana ha un alto tasso di mortalità (4) e una scarsa qualità di vita. Aumentando il numero di dialisi a 5-6 volte a settimana si migliora la qualità di vita, si riduce il rischio cardiovascolare e si prolunga la sopravvivenza rispetto alla dialisi trisettimanale (1-5).

Nel tentativo di migliorare i risultati per questa popolazione di pazienti, alcuni nuovi approcci terapeutici sono stati intrapresi con l'emodialisi. Un aumento della frequenza della dialisi è stato associato a un miglioramento di anemia, ipertrofia ventricolare sinistra, ipertensione, iperfosfatemia, nutrizione e qualità della vita (6-8). Tuttavia, l'accesso a queste modalità di dialisi promettenti è rimasto limitato a causa dell'elevato costo dei trattamenti e del costo del trasporto pazienti. Con questo progetto si va a discutere sul come una riorganizzazione logistica dei trasporti e dei turni di dialisi possa dare un miglioramento qualitativo della vita riducendo 
le comorbilità legate all'insufficienza renale e al trattamento dialitico e aumentando l'aspettativa di vita abbattendo i costi.

A tal fine, nello studio, sono stati arruolati 10 pazienti, i quali, in un primo tempo, sono stati trasportati per eseguire il trattamento dialitico senza una strategia logistica, al fine di confrontarne successivamente i dati ottenuti con simulazioni basate su riorganizzazioni dei trasporti.

L'antinomia emodialisi e abbattimento dei costi ha dato il via alla stesura di questo elaborato, traendo ispirazione dal teorico della Decrescita Serge Latouche, il quale sostiene che, con risorse limitate, una società può solo inventare una logica sociale sostenibile, cambiando valori e concetti, mutando le strutture, rilocalizzando l'economia e la vita e rivedendo nel profondo i modi d'uso dei prodotti. In tal senso, con questo elaborato, si è cercato di valutare se sia possibile minimizzare i costi attraverso una dialisi a basso impatto economico con una riorganizzazione logistica dei trasporti. Latouche la definirebbe "a-crescita" emodialitica, così come si parla di "a-teismo", poiché si tratta di abbandonare una fede, una religione, quella della dialisi ad alto impatto economico trisettimanale, per una a basso impatto economico, daily hemodialysis, individuando, così, degli elementi alternativi per una politica del dopo-sviluppo, migliorando significativamente la salute del paziente e diminuendo le comorbilità e i costi che da questa dipendono (9).

L'obiettivo di questo progetto è quello di:

- migliorare la qualità di vita della persona;

- ridurre i costi di trasporto;

- valutare il risparmio tra i due metodi di trattamento dialisi quotidiana e dialisi trisettimanale con una riorganizzazione logistica dei trasporti.

\section{Materiali e metodi}

Nello studio eseguito all'Ospedale Santa Maria della Misericordia di Perugia sono stati presi in esame 10 pazienti in base alla loro disponibilità.

I pazienti arruolati nello studio sono stati trasportati per eseguire il trattamento díalitico in un centro dialisi ad assistenza continuativa (CAD) senza una strategia logistica, al fine di confrontare successivamente i dati così ottenuti con simulazioni basate su riorganizzazioni logistiche dei trasporti.

Al fine di abbattere i costi di trasporto, si è applicata una strategia di logistica basata su questi assunti:

1) suddivisione degli utenti che abitano nella stessa area urbana in gruppi, in modo da ridurre i mezzi necessari per il trasporto dei pazienti;

2) organizzazione della dialisi di questi pazienti nel medesimo turno dialitico;

3) organizzazione della turnistica dei pazienti;

4) rivalutazione clinica dei pazienti al fine di decidere quali siano trasportabili con mezzi attrezzati e quali con autista;

5) riorganizzazione delle ambulanze e dei pulmini per il tra- sporto dei pazienti;

6) riunione con gli autisti per esortarli al rispetto degli orari.

Per non avere inconvenienti nella tempistica di trasporto, gli autisti dovranno dare l'orario di prelevamento al domicilio al paziente. Si eseguiranno, poi, delle simulazioni e si considereranno due parametri comuni che sono il numero di viaggi e i chilometri totali percorsi.

Per eseguire le simulazioni si è proceduto con tre fasi successive:

1) definizione del campione di utenti; per avere dei valori attendibili si è considerato un numero di utenti significativo, pari a 100 pazienti;

2) localizzazione degli utenti; per ogni utente si è ipotizzata una distanza tra la sua abitazione e l'ospedale, compresa tra 1 chilometro e un valore massimo di 50 chilometri, cioè la massima distanza tra l'ospedale di Perugia e il confine provinciale. Le posizioni delle abitazioni degli utenti e, conseguentemente, le loro distanze dall'ospedale sono state generate in maniera casuale con coordinate GPS cadenti all'interno di un cerchio centrato nell'ospedale di Perugia, con un raggio pari alla massima distanza dall'ospedale di un utente: definiremo "localizzazione" la creazione delle posizioni delle abitazioni degli utenti;

3) raggruppamento; dopo aver localizzato i pazienti, questi sono stati riuniti in gruppi. II raggruppamento ha tenuto conto della capacità dei mezzi di trasporto e, quindi, si è considerata una capacità massima come parametro prefissato per le diverse simulazioni.

Nella prima serie di simulazioni, si è considerata una capacità massima (C) di 8 pazienti, corrispondente a un pulmino di 9 posti comprensivi del conducente. In una seconda serie di simulazioni, la capacità massima $\mathrm{C}$ sarà pari a 5 pazienti, corrispondente a un pulmino di 6 posti comprensivi del conducente. Nell'ultima serie, infine, si è considerata una capacità $C$ pari a 4 utenti, corrispondente a quella di un autoveicolo.

Il parametro sulla base del quale sono stati eseguiti gli accorpamenti dei pazienti negli spostamenti dall'ospedale e per l'ospedale è la distanza dal medesimo e tra i pazienti stessi: sono stati, quindi, considerati, in uno stesso gruppo, fino a 8 , 5 o 4 utenti che risultavano abitare in una stessa zona, rispettivamente.

La massima distanza tra due pazienti, affinché questi possano considerarsi "vicini", è stata fissata a $10 \mathrm{~km}$, tenendo presente la velocità media del veicolo nel ciclo urbano, il carico dei pazienti nel veicolo e il ritorno in ospedale.

Nel calcolo della lunghezza dei percorsi, per ciascun gruppo si è considerato un "worst case", sommando la distanza massima possibile tra i pazienti alla media della loro distanza dall'ospedale. Nel calcolo del numero di corse, si è considerato che ogni gruppo corrisponde a un viaggio. 


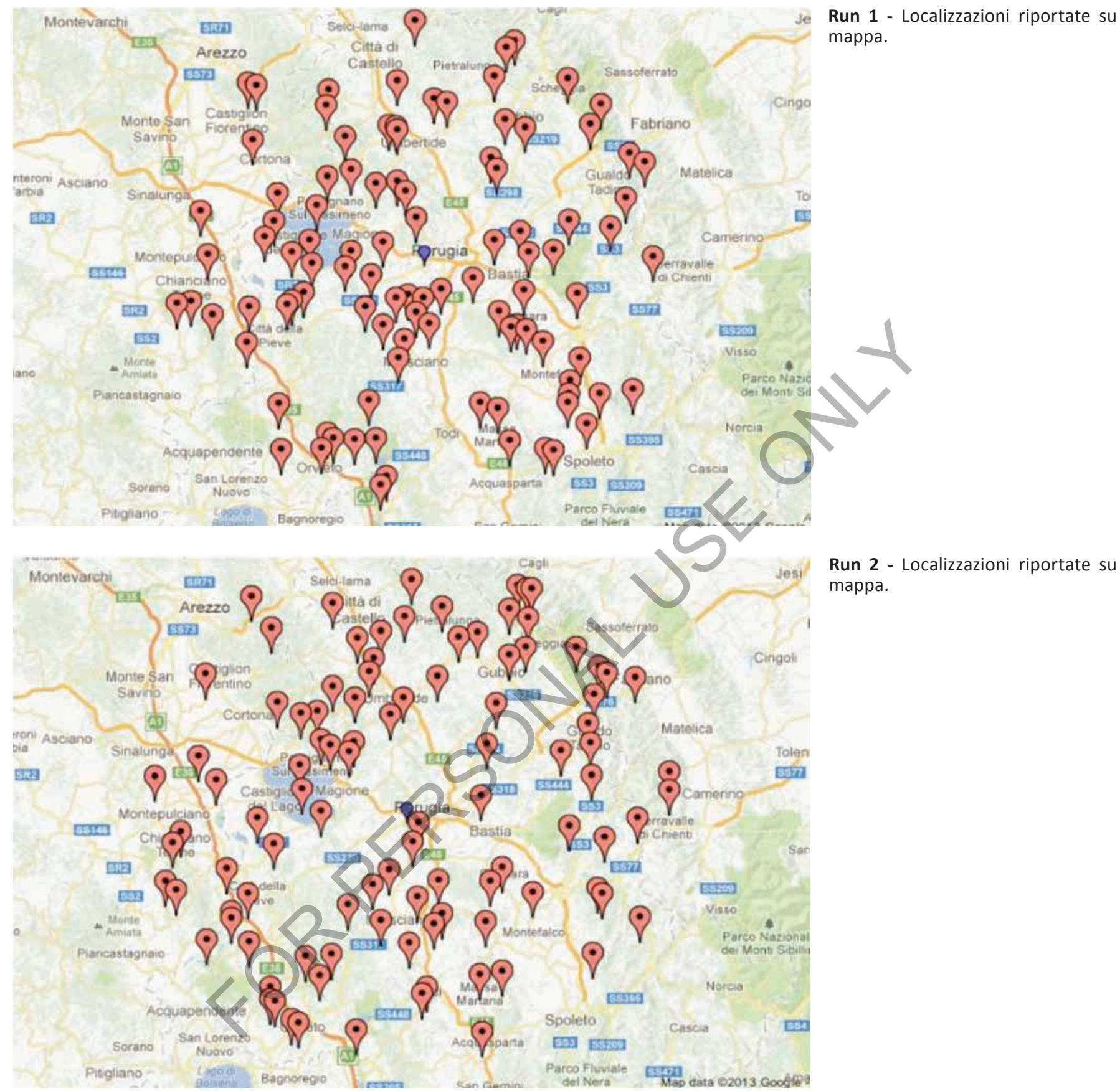

Per ogni valore di capacità $\mathrm{C}$, sono state generate 10 prove, che chiameremo Run, con un campione di 100 pazienti ciascuna, ottenendo un valore medio dei risultati delle prove stesse. L'elaborazione di tali dati è stata eseguita tramite la Visual Basic Application (VBA).

Il software per il calcolo delle distanze è stato sviluppato in due punti:

1) calcolo della distanza tra tutti i pazienti in tutte le possibili combinazioni, cioè calcolo del prodotto cartesiano delle distanze tra gli utenti;

2) raggruppamenti: il raggruppamento ha, come assunzioni, due variabili:

- distanza massima tra due pazienti: questa variabile indicherà al software qual è la massima distanza tra due pazienti per poter far parte dello stesso gruppo;

- massimo raggruppamento: il numero massimo di pazienti all'interno dello stesso gruppo. 


\section{Strategia di abbattimento dei costi di trasporto}

Al fine di abbattere i costi di trasporto, si pensa di applicare una strategia di logistica basata sul raggruppamento per zone dei pazienti e si prevede, all'atto pratico, una contrattazione dei prezzi per chilometro o per numero di corse verso il fornitore del servizio di trasporto. Questo metodo consente di abbattere i costi che possono essere ricompresi attraverso dei costi variabili diretti e, quindi, riassorbiti da una dialisi a basso costo.

La misura del costo deve essere eseguita prendendo in considerazione parametri normalizzati per tutte le tipologie di trasporto; si sono, pertanto, considerati due parametri comuni, che sono il numero di viaggi e i chilometri totali percorsi. Attualmente, le spese dei trasporti sono basate o sul chilometraggio o sulla quantità di pazienti trasportati e, attualmente, in Italia, non esiste una legge nazionale che orienti questa problematica.

Per eseguire le simulazioni, si è, quindi, proceduto con 3 fasi successive:

1) definizione del campione di utenti.

Per avere dei valori attendibili è stato considerato un numero di utenti significativo, pari a 100 pazienti;

2) localizzazione degli utenti.

Per ogni utente è stata ipotizzata una distanza tra la sua abitazione e l'ospedale compresa tra 1 chilometro ê un valore massimo. La massima distanza dall'ospedale di un utente è stata considerata pari a 50 chilometri, all'incirca la massima distanza tra l'ospedale di Perugia e il confine provinciale. Le posizioni delle abitazioni degli utenti e, conseguentemente, le loro distanze dall'ospedale sono state generate in maniera casuale con coordinate GPS cadenti all'interno di un cerchio centrato nell'ospedale di Perugia, con un raggio pari alla massima distanza dall'ospedale di un utente: definiamo "localizzazione" la creazione delle posizioni delle abitazioni degli utenti.

Le immagini mostrano i risultati di alcune localizzazioni riportate su mappa.

I grafici delle Figure 1, 2 e 3 mostrano la composizione che si è ottenuta per i gruppi in ciascuna delle prove eseguite: i raggruppamenti numerosi, anche quando siano in basso numero, consentono di ottenere un notevole risparmio nei parametri presi in considerazione per valutare il costo.

I seguenti grafici (Fig. 4) riportano i risultati in forma percentuale, evidenziando il risparmio in termini di chilometri percorsi e in numero di corse che si ottengono applicando una strategia di logistica. Come si vede, con una capacità $\mathrm{C}=8$, si ha un risparmio medio di circa il 58\% in termini di chilometraggio e del $70 \%$ in termini di corse effettuate, con C = 5 il risparmio medio è pari al $52.4 \%$ per i chilometri percorsi e al $62 \%$ per le corse effettuate e con $\mathrm{C}=4$ si ottiene un risparmio medio uguale al $49 \%$ sul chilometraggio e al $57 \%$ sul numero di corse.

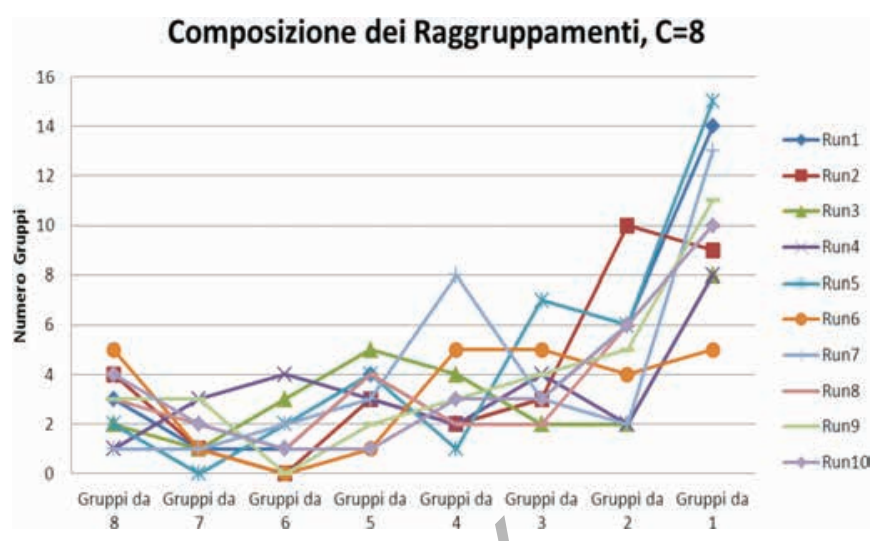

Fig. 1 - Distribuzione dei gruppi per veicoli con massima capacità pari a 8 .

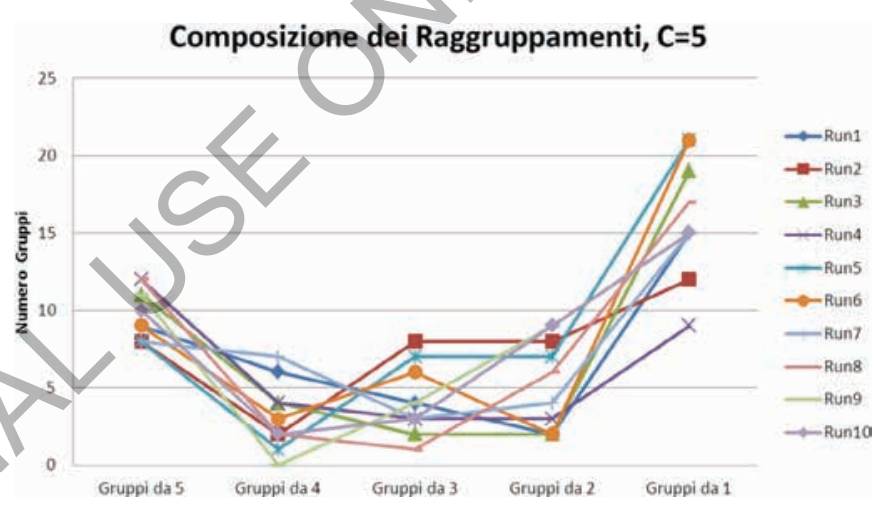

Fig. 2 - Distribuzione dei gruppi per veicoli con massima capacità pari a 5 .

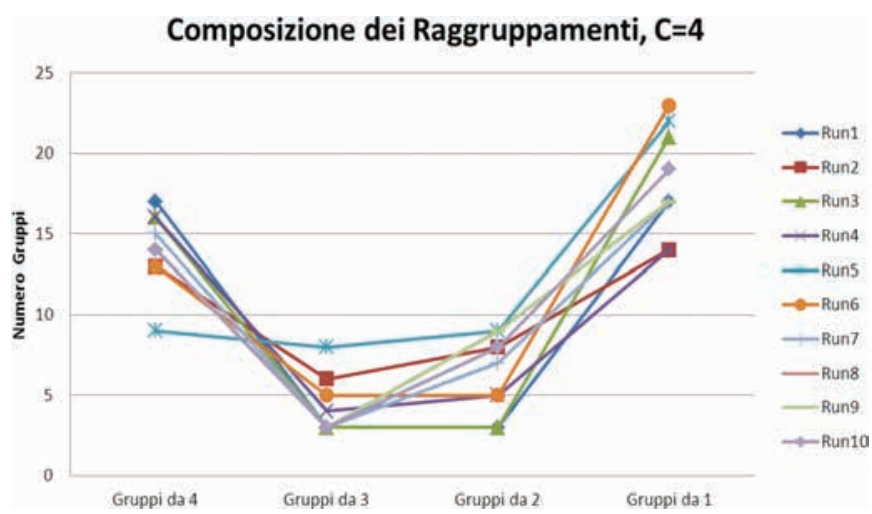

Fig. 3 - Distribuzione dei gruppi per veicoli con massima capacità pari a 4.

\section{Confronto tra emodialisi quotidiana ed emodialisi trisettimanale: simulazioni con dati statistici}

Nei seguenti grafici si riporta il confronto tra il costo settimanale della modalità di trasporto con Strategia di Logistica per la dialisi quotidiana e il costo settimanale della modalità di tra- 


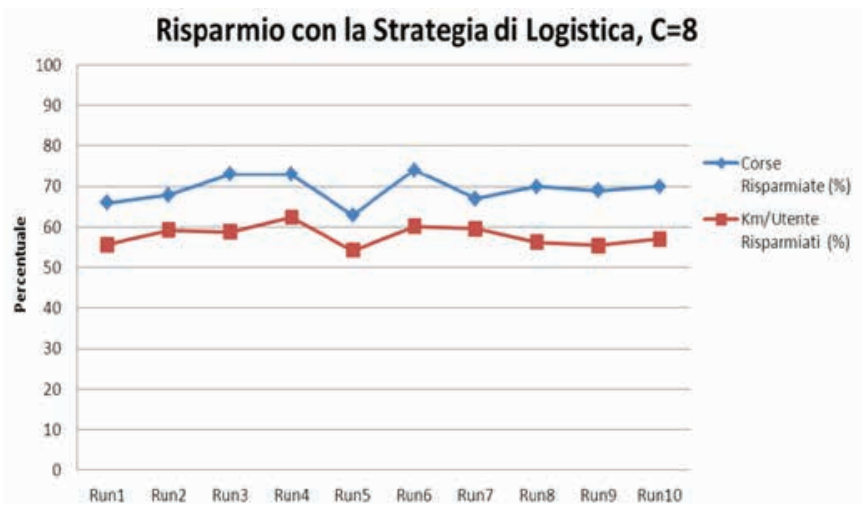

Fig. 4 - Capacità $C=8$ si ha un risparmio medio di circa il 58\% in termini di chilometraggio e del $70 \%$ in termini di corse effettuate .

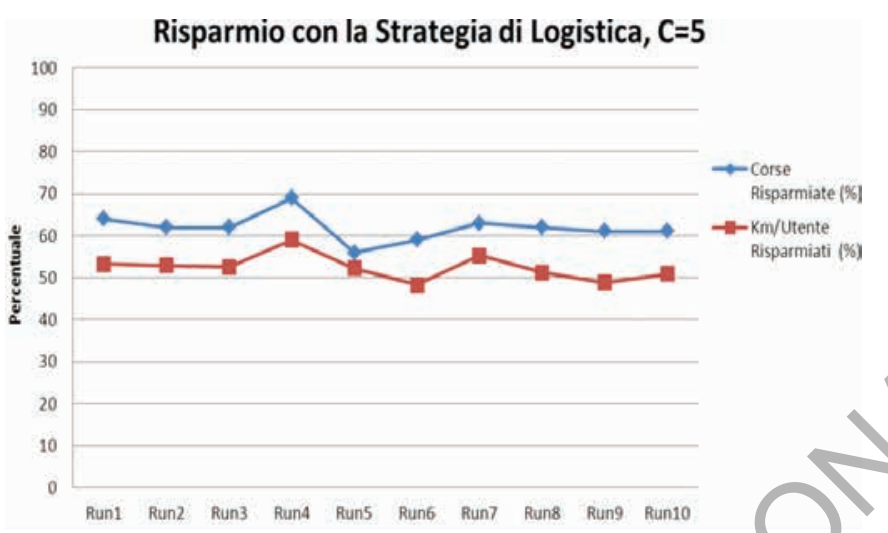

Fig. 5 - $C=5$ il risparmio medio è pari al 52,4\% per i chilometri percorsi e al $62 \%$ per le corse effettuate.

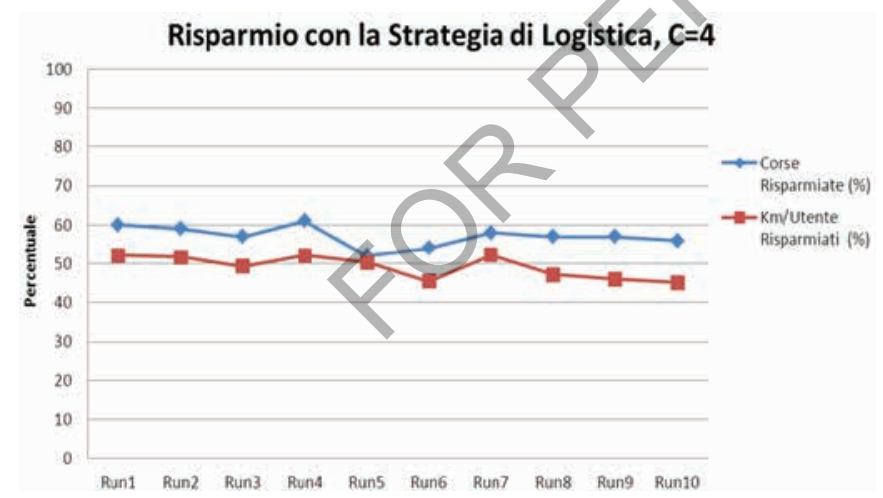

Fig. 6 - $C=4$ si ottiene un risparmio medio uguale al $49 \%$ sul chilometraggio e al $57 \%$ sul numero di corse.

sporto senza Strategia di Logistica per la dialisi trisettimanale.

I grafici delle Figure 5, 6 e 7 mostrano nelle ascisse le diverse prove e nelle ordinate il costo chilometrico settimanale ( $\mathrm{km}$ percorsi a settimana), applicando la strategia di logistica a una dialisi quotidiana (6 giorni su 7) contro il costo chilometrico settimanale di una dialisi trisettimanale (3 giorni su 7) senza strategia di logistica.

I grafici delle Figure 8, 9 e 10 mostrano nelle ascisse le diverse prove e nelle ordinate il numero di corse settimanali, applicando la strategia di logistica a una dialisi quotidiana (6 giorni su 7) contro il numero di corse settimanali di una dialisi trisettimanale (3 giorni su 7) senza strategia di logistica.

II grafico di Figura 11 analizza il numero di corse settimanali con c 5 di una dialisi quotidiana con strategia di logistica a confronto con una dialisi trisettimanale senza strategia di logistica.

La Figura 12 analizza la dialisi quotidiana con strategia di logistica e la dialisi trisettimanale senza strategia di logistica, con c 4 prendendo in esame il numero di corse settimanali su 10 prove eseguite ed ogni prova conteneva 100 pazienti.

Come si vede, applicando la strategia di logistica, il costo di trasporto settimanale della dialisi quotidiana è non solo paragonabile ma persino inferiore al costo di trasporto settimanale della dialisi trisettimanale, sia in termini di chilometri percorsi pro capite che in termini di numero di corse.

\section{Caso reale}

È stata simulata l'applicazione della Strategia di Logistica a un gruppo di 10 pazienti attualmente in cura presso l'ospedale S. Maria della Misericordia di Perugia con dialisi quotidiana, con raggruppamenti di diversa capacità.

La Figura 13 mostra il caso reale,cioè un gruppo di 10 pazienti che sono in cura presso l'ospedale Santa Maria della Misericordia.

Il grafico della Figura 14 identifica la composizione che si è ottenuta per i gruppi nel caso reale preso in esame in Figura 13.

II grafico della Figura 15 mostra nelle ascisse le diverse prove e nelle ordinate il numero di chilometri settimanali percorsi, applicando la strategia di logistica a una dialisi quotidiana (6 giorni su 7) contro il numero di corse settimanali di una dialisi trisettimanale (3 giorni su 7) senza strategia di logistica.

II grafico della Figura 16 mostra nelle ascisse le diverse prove e nelle ordinate il numero di corse settimanali, applicando la strategia di logistica a una dialisi quotidiana (6 giorni su 7) contro il numero di corse settimanali di una dialisi trisettimanale (3 giorni su 7) senza strategia di logistica.

II grafico ottenuto (Fig. 17) mostra un risparmio in termini di numero di corse del $60 \%$ e di chilometri percorsi pari al $30 \%$ circa, dunque in linea con le simulazioni precedenti.

\section{Discussione}

La dialisi, a tutt'oggi, rimane il trattamento di elezione per I'insufficienza renale cronica (10). Esistono vari schemi dialitici che vengono praticati per i benefici che apportano e questi sono: emodialisi notturna, emodialisi lunga notturna ed emodialisi diurna breve (in genere 2 ore) per sei giorni a settimana, ma lo schema più convenzionalmente usato è quello eseguito per 3 volte a settimana per 4 ore.

Alcuni studi hanno evidenziato, però, che lo schema trisetti- 


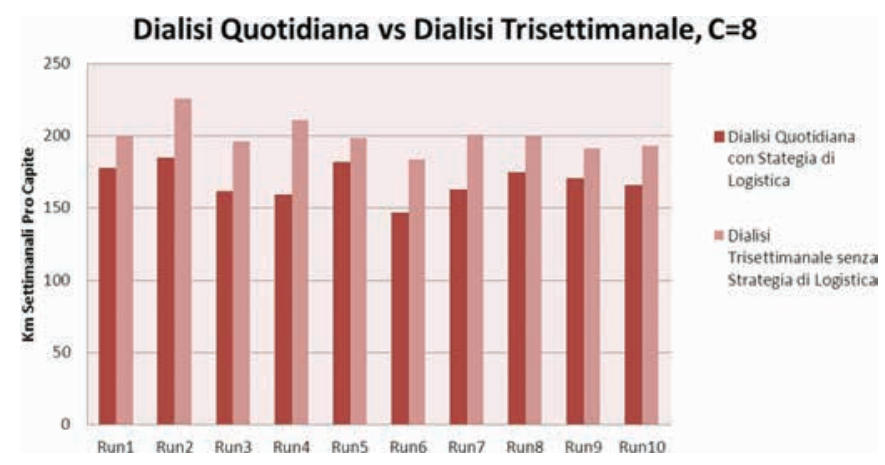

Fig. 7 - II grafico mostra nelle ascisse le diverse prove e nelle ordinate il costo chilometrico settimanale ( $\mathrm{km}$ percorsi a settimana) applicando la strategia di logistica a una dialisi quotidiana (6 giorni su 7) contro il costo chilometrico settimanale di una dialisi trisettimanale (3 giorni su 7) senza strategia di logistica.

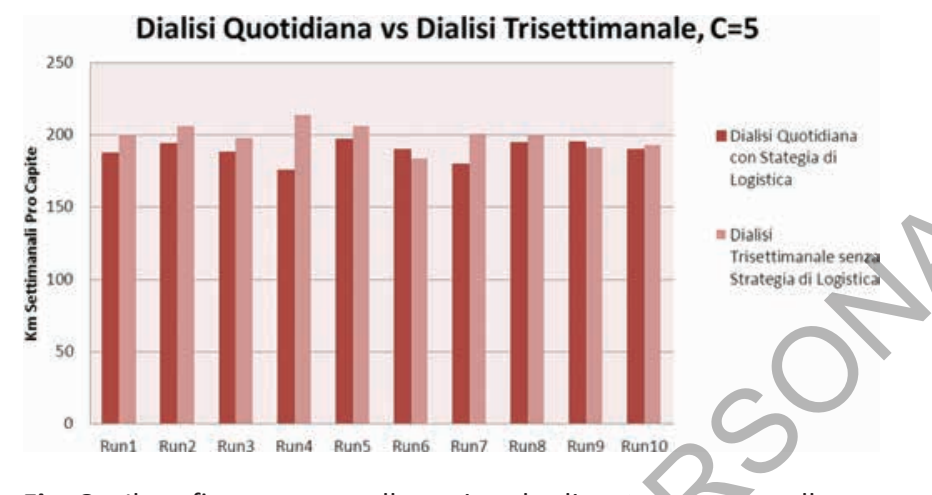

Fig. 8 - II grafico mostra nelle ascisse le diverse prove e nelle ordinate il costo chilometrico settimanale ( $\mathrm{km}$ percorsi a settimana) applicando la strategia di logistica a una dialisi quotidiana (6 giorni su 7) contro il costo chilometrico settimanale di una dialisi trisettimanale (3 giorni su 7) senza strategia di logistica.
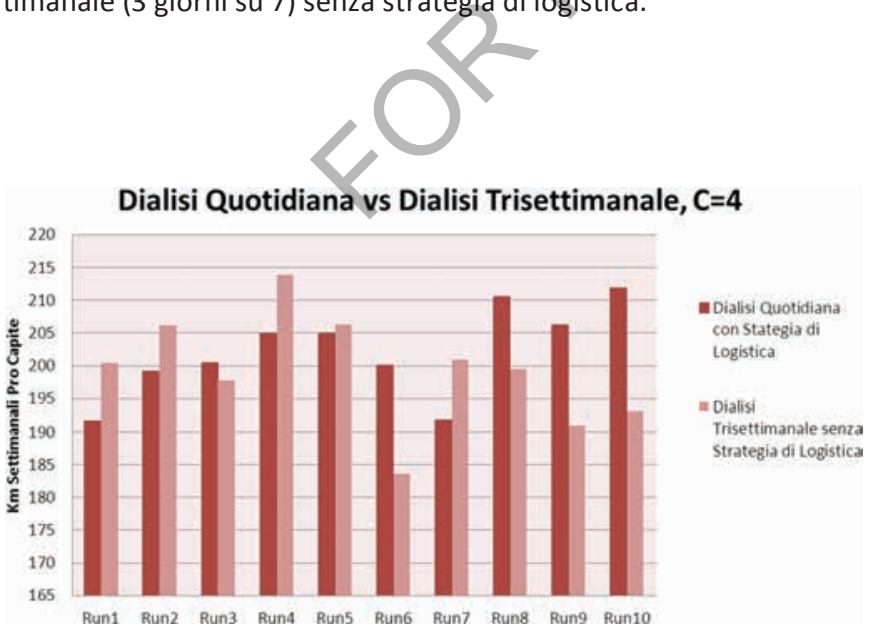

Fig. 9 - II grafico mostra nelle ascisse le diverse prove e nelle ordinate il costo chilometrico settimanale ( $\mathrm{km}$ percorsi a settimana) applicando la strategia di logistica a una dialisi quotidiana (6 giorni su 7) contro il costo chilometrico settimanale di una dialisi trisettimanale (3 giorni su 7) senza strategia di logistica.

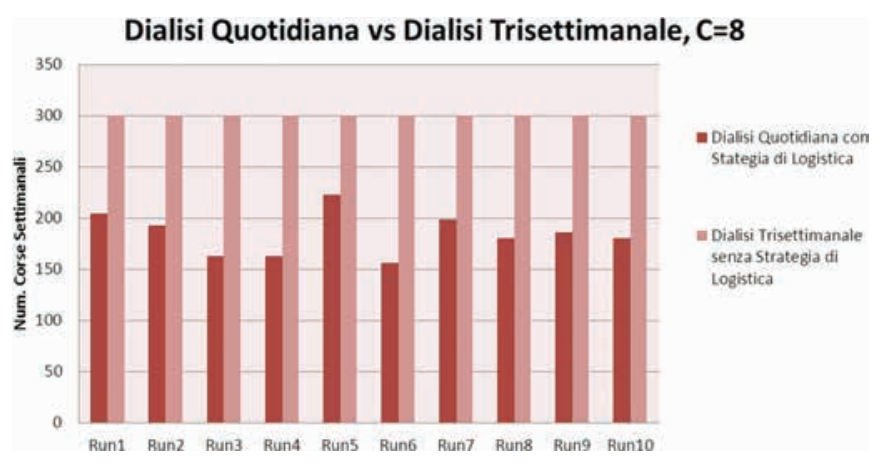

Fig. 10 - Il grafico mostra nelle ascisse le diverse prove e nelle ordinate il numero corse settimanali applicando la strategia di logistica a una dialisi quotidiana (6 giorni su 7) contro il numero di corse settimanali di una dialisi trisettimanale (3 giorni su 7) senza strategia di logistica.
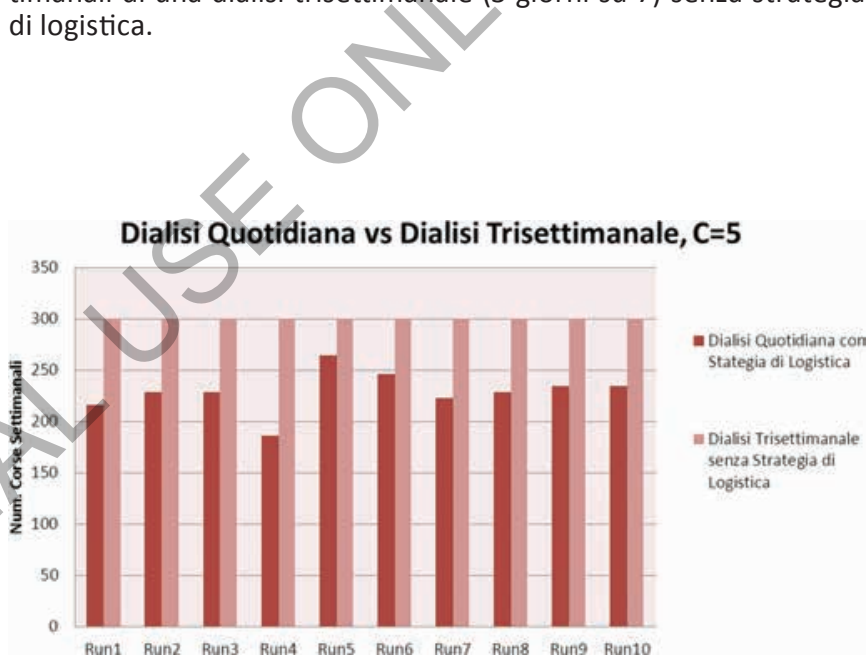

Fig. 11 - Il grafico mostra nelle ascisse le diverse prove e nelle ordinate il numero corse settimanali applicando la strategia di logistica a una dialisi quotidiana ( 6 giorni su 7) contro il numero di corse settimanali di una dialisi trisettimanale (3 giorni su 7) senza strategia di logistica.

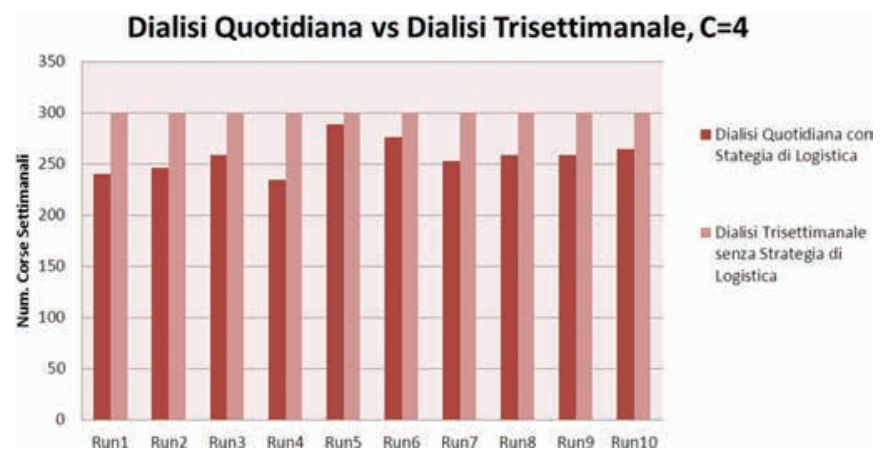

Fig. 12 - Il grafico mostra nelle ascisse le diverse prove e nelle ordinate il numero corse settimanali applicando la strategia di logistica a una dialisi quotidiana (6 giorni su 7) contro il numero di corse settimanali di una dialisi trisettimanale (3 giorni su 7) senza strategia di logistica. 


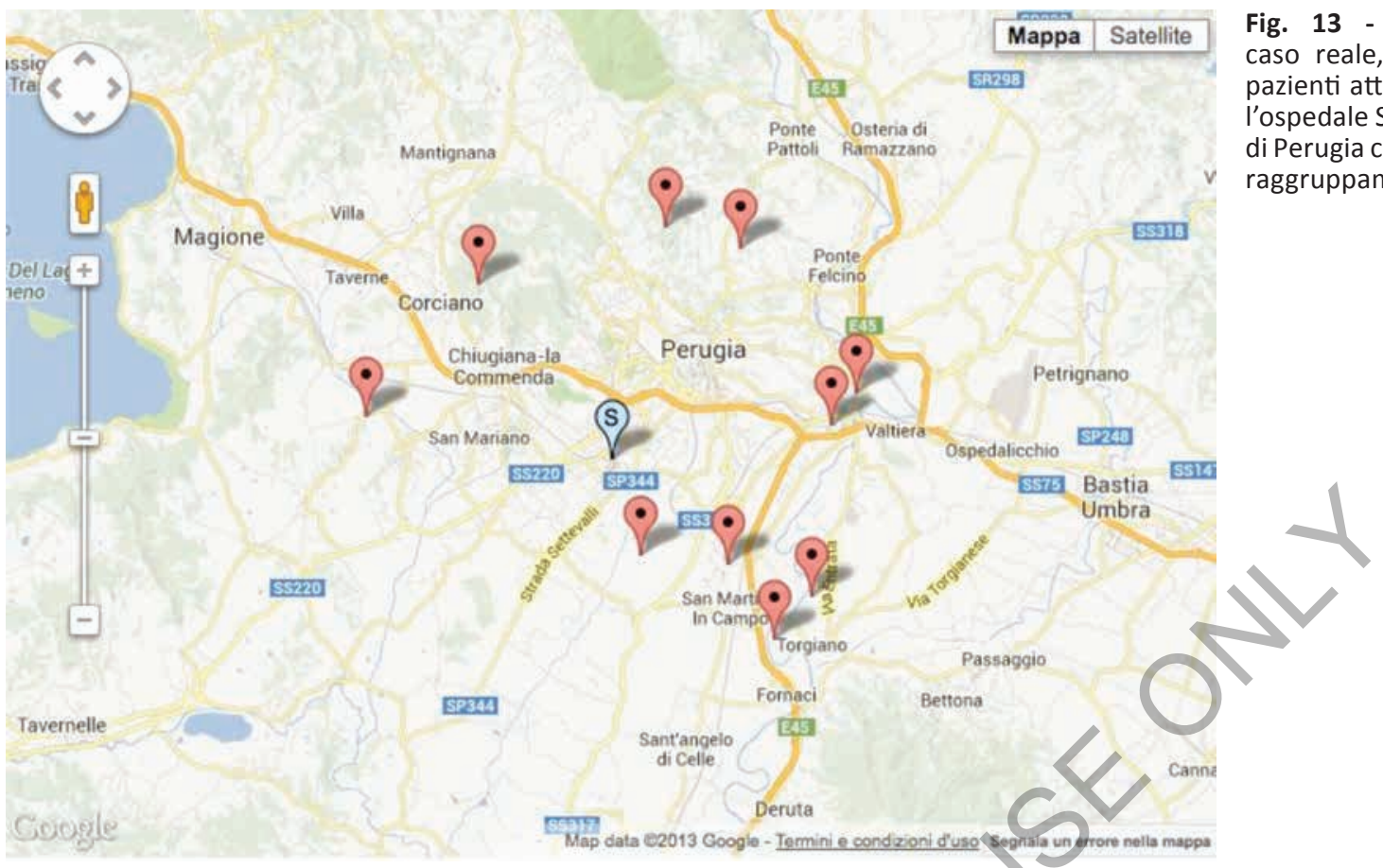

Composizione dei Raggruppamenti

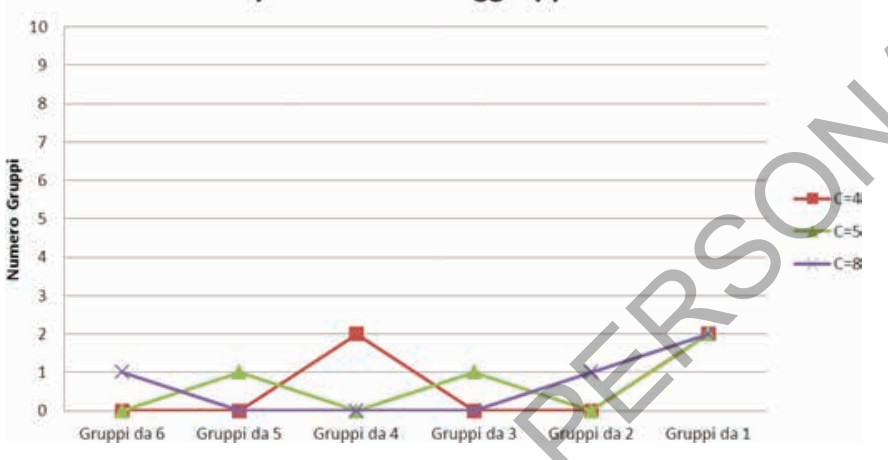

Fig. 14 - II grafico mostra la composizione che si è ottenuta per i gruppi nel caso reale.

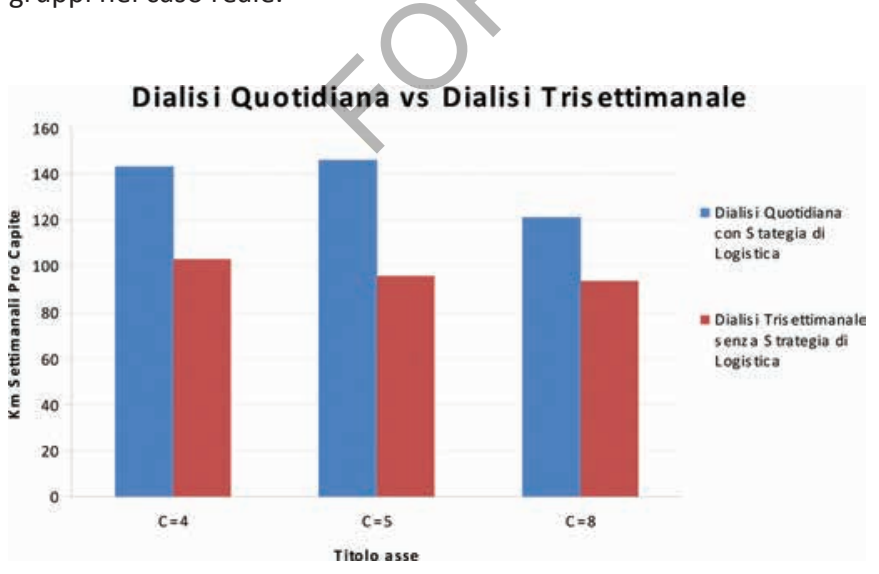

Fig. 15 - Il grafico mostra nelle ascisse le diverse prove e nelle ordinate il numero di chilometri settimanali percorsi applicando la strategia di logistica a una dialisi quotidiana (6 giorni su 7) contro il numero di corse settimanali di una dialisi trisettimanale ( 3 giorni su 7) senza strategia di logistica.
Dialisi Quotidiana vs Dialisi Tris ettimanale

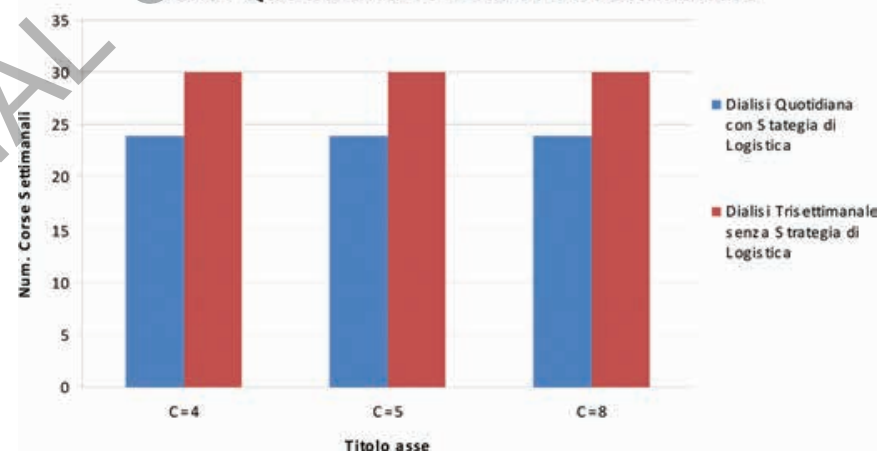

Fig. 16 - II grafico mostra nelle ascisse le diverse prove e nelle ordinate il numero corse settimanali applicando la strategia di logistica a una dialisi quotidiana (6 giorni su 7) contro il numero di corse settimanali di una dialisi trisettimanale (3 giorni su 7) senza strategia di logistica.

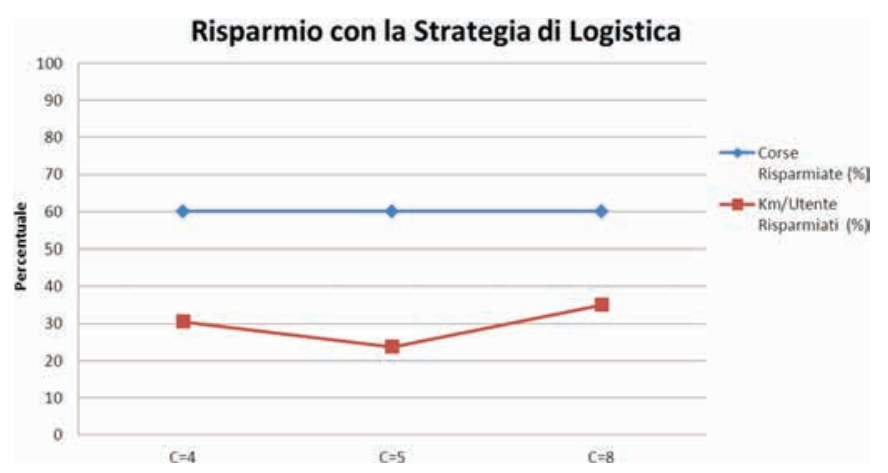

Fig. 17 - Il grafico dimostra un risparmio in termini di numero di corse del $60 \%$ e di chilometri percorsi pari al $30 \%$ circa, dunque in linea con le simulazioni precedenti. 
manale non ha portato un cambiamento significativo delle comorbilità e della sopravvivenza dei pazienti, evidenziando, inoltre, che la dialisi più vicina alla fisiologicità del paziente è una dialisi breve eseguita per sei giorni a settimana per due ore, che riduce $\mathrm{i}$ costi sia dei farmaci assunti che dei ricoveri dati dalle comorbilità.

Tale metodica, anche se ben tollerata dal paziente, con la restituzione di un buon vigore fisico e con l'abbattimento delle comorbilità, incontra delle problematiche a livello dei costi, la maggior parte dei quali è assorbita dai trasporti. II sistema di riorganizzazione logistica dei trasporti proposto si prefigge lo scopo di ridurre i costi suddividendo gli utenti che abitano nella stessa zona in modo da ridurre i mezzi necessari per il trasporto dei pazienti. La sperimentazione è stata eseguita in un centro dialisi ad assistenza continuativa (CAD), quindi i pazienti possono essere complessi e in condizioni cliniche a rischio; è stata eseguita, perciò, una valutazione critica delle varianti che possono entrare in gioco "come dimostrato nelle simulazioni" alterando, così, significativamente i costi. Ovviamente, una ristrutturazione logistica dei trasporti in un centro dialisi ad assistenza limitata (CAL) risulta più economica e di più facile attuazione per il fatto che i pazienti da sottoporre al trattamento sono selezionati e in condizioni cliniche di bassa complessità, quindi non impegnano mezzi attrezzati come "ambulanze" o solo in rari casi.

Lo studio, inoltre, è stato condotto su un numero di pazienti ristretto per non influire negativamente sul budget se la sperimentazione non avesse dato i risultati sperati, ma le prove ottenute dei casi reali hanno dimostrato un risparmio in termini di numero di corse del $60 \%$ e di chilometri percorsi pari al $30 \%$ circa, dunque in linea con le varie simulazioni effettuate, evidenziando come questa variabile dei trasporti, se ben codificata e strutturata, possa produrre un cambiamento positivo nelle prospettive future della dialisi.

\section{Conclusioni}

Il presente studio ha avuto come obiettivo quello di mettere in evidenza la differenza dei costi e dei benefici della dialisi quotidiana e di quélla trisettimanale analizzando due tipi di trasporto ( $\mathrm{km}$ percorsi e numero di corse) per valutare quale metodo implementare nelle varie realtà.

Dai dati raccolti in letteratura e dal risultato delle simulazioni in cui si è ipotizzata una gestione logistica con vari profili di distribuzione dei pazienti nel territorio regionale umbro, si è evinto che, sebbene, in apparenza, i costi di un'emodialisi standard quotidiana risultino superiori rispetto a quelli della trisettimanale, un'accorta gestione della spesa legata al trasporto dei pazienti abbatte il costo, riportandolo a valori paragonabili a quelli di un'emodialisi trisettimanale. L'impiego di questo nuovo sistema di riorganizzazione logistica dei trasporti può abbattere quel muro, che sembrerebbe insormontabile, dei costi inerenti sia ai trasporti che ai farmaci e, non meno importante, ai ricoveri dati dalle comorbilità.

\section{Ringraziamenti}

Dedico un ringraziamento per la stesura di questo lavoro all'amico Giuseppe Quintaliani non solo per lo stimolo alla stesura e la revisione ma per essere mio insegnante, da me odiato ed amato: odiato perché ha distrutto senza pietà i muri delle mie certezze; amato perché, cosi facendo, mi ha condotto per mano ad immergermi nella luce di altri orizzonti.

\section{Disclosures}

Financial support: No financial support was received for this submission.

Conflict of interest: The authors have no conflict of interest.

\section{Bibliografia}

1. Culleton BF, Asola MR. The impact of short daily and nocturnal hemodialysis on quality of life, cardiovascular risk and survival. J Nephrol. 2011;24(4):405-15.

2. Grassmann A, Gioberge S, Brown G. ESRD patients in 2004: global overview of patient numbers, treatment modalities and associated trends. Nephrol Dial Trasplant. 2005;20:2587-93.

3. Meinero S, Tesio E, Bainotti $S$, et al. Valutazione della qualità di vita dei dializzati del cuneese. [Quality of life assessment in dialyzed patients in the Cuneo area]. G Ital Nefrol. 2011;28(1): 72-9.

Foley RN, Parfrey PS, Sarnak MJ. Epidemilogy of cardiovascular disease in chronic renal disease. J Am Soc Nephrol. 1998;9(12 Suppl.):s16-23.

5. Sghirlanzoni MC, Mingardi G. L'emodialisi lunga e/o quotidiana: solosperanze oqualche certezza? Una dialisi perpochio una reale alternativa? [Daily/nocturnal hemodialysis: only for the happy few or a real alternative for many?]. G Ital Nefrol. 2008;25(2): 192-202.

6. Ayus J, Mizani M, Achinger S, et al. Effects of short daily versus conventional hemodialysis on left ventricular hypertrophy and inflammatory markers: a prospective controlled study. J Am Soc Nephrol. 2005;16:2778-88.

7. Fagugli RM, Reboldi G, Quintaliani G, et al. Short daily hemodialysis. Blood pressure control and left ventricular reduction in hypertensive hemodialysis patients. Am J Kidney Dis. 2001;38:371-6.

8. Fagugli RM, Pasini P, Pasticci F, et al. Effect of short daily hemodialysis and extended standard hemodialysis on blod pressare and cardiac hypertrophy: a comparative study. J Nephrol. 2006;19:77-83.

9. Latouche S. La scommessa della decrescita. Milano Giangiacomo Feltrinelli Editore. 2007;98:11-2.

10. EDTNA/ERCA. Insufficienza renale cronica: una guida per la pratica clinica (stadi 1-3). In: Altea Mahon, Karen Jenkins. Insufficienza renale cronica (stadi 1-3): una guida per la pratica clinica. Switzerland: Tipografia Stigraf S.R.L.-Viadana MN. 2008;22-3. 